\title{
Thrombocyte Inhibition Restores Protective Immunity to Mycobacterial Infection in Zebrafish
}

\author{
Elinor Hortle, ${ }^{1,2}$ Khelsey E. Johnson, ${ }^{3}$ Matt D. Johansen, ${ }^{1}$ Tuong Nguyen, ${ }^{1}$ Jordan A. Shavit, ${ }^{4}$ Warwick J. Britton, ${ }^{1,2}$ David M. Tobin, ${ }^{3}$ and \\ Stefan H. Oehlers ${ }^{1,2}, \odot$ \\ ${ }^{1}$ Tuberculosis Research Program Centenary Institute, The University of Sydney, Camperdown, Australia; ${ }^{2}$ The University of Sydney, Central Clinical School and Marie Bashir Institute, Camperdown, \\ Australia; ${ }^{3}$ Department of Molecular Genetics and Microbiology, Duke University School of Medicine, Durham, North Carolina; ${ }^{4}$ Department of Pediatrics and Cellular and Molecular Biology \\ Program, University of Michigan, Ann Arbor
}

Background. Infection-induced thrombocytosis is a clinically important complication of tuberculosis infection. Recent studies have highlighted the utility of aspirin as a host-directed therapy modulating the inflammatory response to infection but have not investigated the possibility that the effect of aspirin is related to an antiplatelet mode of action.

Methods. In this study, we utilize the zebrafish-Mycobacterium marinum model to show mycobacteria drive host hemostasis through the formation of granulomas. Treatment of infected zebrafish with aspirin markedly reduced mycobacterial burden. This effect is reproduced by treatment with platelet-specific glycoprotein IIb/IIIa inhibitors demonstrating a detrimental role for infectioninduced thrombocyte activation.

Results. We find that the reduction in mycobacterial burden is dependent on macrophages and granuloma formation, providing the first in vivo experimental evidence that infection-induced platelet activation compromises protective host immunity to mycobacterial infection.

Conclusions. Our study illuminates platelet activation as an efficacious target of aspirin, a widely available and affordable hostdirected therapy candidate for tuberculosis.

Keywords. clotting; hemostasis; innate immunity; mycobacterial infection.

Mycobacterium tuberculosis causes approximately 2 million deaths each year [1]. The increasing incidence of drug-resistant tuberculosis (TB) urgently requires the development of novel therapeutic strategies. Pathogenic mycobacteria co-opt host pathways to establish persistent infection, and inhibition of this subversion with host-directed therapies (HDTs) can reduce the severity of infection in animal models. We have recently shown that mycobacteria induce host angiogenesis and increase host vascular permeability; blockade of either of these processes reduced the growth and spread of bacteria $[2,3]$.

Thrombocytosis is a recognized biomarker for advanced TB, and infection is often accompanied by a hyper-coagulable state, resulting in increased risk of deep vein thrombosis (DVT) and stroke $[4,5]$. Recent evidence hints that mycobacteria may drive

Received 3 January 2019; editorial decision 4 March 2019; accepted 7 March 2019; published online March 16, 2019

Presented in part: Keystone TB Meeting, January 2019, Banff, AB, Canada and April 2018, Whistler, BC, Canada; Australia and New Zealand Zebrafish Meeting January 2018, Sydney, NSW, Australia and 2017, Auckland, New Zealand; China Australia TB Immunology Meeting December 2017, Zhuhai, China and October 2018, Sydney, NSW, Australia; Lorne Infection and Immunity Meeting, Lorne, VIC, Australia.

Correspondence: S. H. Oehlers, PhD, Tuberculosis Research Program, Centenary Institute, Locked Bag 6, Newtown NSW 2042, Australia (stefan.oehlers@sydney.edu.au).

\section{The Journal of Infectious Diseases ${ }^{\circledR} \quad$ 2019;220:524-34}

(c) The Author(s) 2019. Published by Oxford University Press for the Infectious Diseases Society of America. All rights reserved. For permissions, e-mail: journals.permissions@oup.com. DOl: 10.1093/infdis/jiz110 hemostasis, and that hemostasis may aid their growth. For example, cell wall components from $M$ tuberculosis can induce coagulation via macrophage expression of tissue factor [6]. In mice and humans, markers of platelet activation are upregulated during $M$ tuberculosis infection [7, 8], and in vitro exposure to platelets increases the conversion of infected macrophages into cells permissive for bacterial growth $[7,9]$. To date, the pathogenic roles of hemostasis have not been studied in an intact in vivo model of mycobacterial infection.

In this study, we used the zebrafish- $M$ marinum model to investigate the role of host thrombocytes in mycobacterial infection. We present evidence that although coagulation and thrombocyte activation are both driven by mycobacteria, it is only infection-induced activation of thrombocytes that specifically compromises protective immunity through direct thrombocyte-macrophage interactions.

\section{METHODS}

\section{Zebrafish Husbandry}

Adult zebrafish were housed at the Garvan Institute of Medical Research Biological Testing Facility (St. Vincent's Hospital AWC Approval 1511), and embryos were produced by natural spawning for infection experiments at the Centenary Institute (Sydney Local Health District AWC Approval 2016-022). Zebrafish embryos were obtained by natural spawning, and embryos were raised at $28^{\circ} \mathrm{C}$ in $\mathrm{E} 3$ media. 


\section{Zebrafish Lines}

Wild-type (WT) zebrafish are the TAB background. Transgenic lines are as follows: $\operatorname{Tg}(f a b p 10 a: f g b-E G F P)^{m i 4001}$ referred to as $T g(f a b p 10 a: f g b-E G F P)$ [10], $T g$ (-6.0itga2b:eGFP) ${ }^{l a 2}$ referred to as $\operatorname{Tg}(c d 41: E G F P)$ [11], and $\operatorname{Tg}$ (mfap4:tdTomato $)^{x t 12}$ referred to as $\mathrm{Tg}$ (mfap4:tdTomato) [12]. Mutant allele $f g a^{m i}$ contains a 26-base pair (bp) insertion in the fibrinogen alpha chain gene [13].

\section{Infection of Zebrafish Embryos}

Aliquots of single-cell suspensions of midlog-phase Mycobacterium marinum $\mathrm{M}$ strain and $\triangle \mathrm{ESX} 1 M$ marinum were frozen at $-80^{\circ} \mathrm{C}$ for use in infection experiments. Bacterial aliquots were thawed and diluted with phenol red dye $(0.5 \%$ $\mathrm{w} / \mathrm{v})$. A total of $10-15 \mathrm{~nL}$ was injected into the caudal vein or trunk of M-222 (tricaine)-anaesthetized, 30- to 48-hour postfertilization embryos resulting in a standard infectious dose of $\sim 400$ fluorescent $M$ marinum. Embryos were recovered into E3 supplemented with $0.036 \mathrm{~g} / \mathrm{L}$ phenylthiourea, housed at $28^{\circ} \mathrm{C}$, and imaged on day 5 of infection unless otherwise stated.

\section{Infection of Zebrafish Adults}

Three- to twelve-month-old zebrafish were infected with $\sim 200$ colony-forming units $M$ marinum by intraperitoneal injection as previously described [3]. Bacterial burden was quantified by $M$ marinum 16 s quantitative polymerase chain reaction as previously described [14].

\section{Drug Treatments}

Fish were treated with vehicle control (dimethyl sulfoxide [DMSO] or water as appropriate), $10 \mu \mathrm{g} / \mathrm{mL}$ aspirin, $20 \mu \mathrm{g} / \mathrm{mL}$ tirofiban, $10 \mu \mathrm{M}$ eptifibatide, or $5 \mu \mathrm{M}$ warfarin. Drugs were replaced every second day starting immediately (embryos) or 1 week postinfection (adults), unless otherwise stated.

\section{Tail Wound Thrombosis Assay}

Three-day postfertilization (DPF) embryos were treated overnight with antiplatelet drugs. They were anaesthetized, and then a small amount of their tail was removed with a scalpel. Embryos were imaged 4 hours postwounding, and the number of green fluorescent protein (GFP)-positive cells within $100 \mu \mathrm{m}$ of the cut site was counted.

\section{Imaging}

Live zebrafish embryos were anaesthetized in M-222 (Tricaine) and mounted in 3\% methylcellulose for static imaging on a Leica M205FA or DM6000B fluorescence stereomicroscope. Image analysis was carried out with Image J Software Version $1.51 \mathrm{j}$ using fluorescent pixel counts and intensity measurements as previously described [15].

Video and time-lapse imaging was carried out on anaesthetized embryos mounted in $0.75 \%$ low melting point agarose on a Leica M205FA or Deltavision Elite fluorescence microscope. Video editing was carried out with Image J Software Version $1.51 \mathrm{j}$ and iMovie. Thrombocyte tracks were generated using the TrackMate plugin for Image J [16].

\section{Axenic Culture}

A midlog culture of fluorescent $M$ marinum was diluted 1:100 and aliquoted into 96-well plates for drug treatment. Cultures were maintained at $28^{\circ} \mathrm{C}$ in a static incubator and bacterial fluorescence was measured in a plate reader.

\section{Morpholinos}

Embryos were injected at the single-cell stage with $1 \mathrm{pmol} \mathrm{cMPL}$ (5'-CAGAACTCTCACCCTTCAATTATAT-3') or control morpholino (5'-CCTCTTACCTCAGTTACAATTTATA-3').

\section{Clodronate Liposome Injections}

Larvae were injected at 3 days postinfection (DPI) (4 DPF) with $10 \mathrm{~nL}$ of $5 \mathrm{mg} / \mathrm{mL}$ clodronate liposomes or $5 \mathrm{mg} / \mathrm{mL}$ phosphatebuffered saline vehicle liposomes by caudal vein injection.

\section{Oil Red 0}

Oil Red O lipid staining on whole-mount embryos was performed and analyzed as previously described $[17,18]$. In brief, embryos were individually imaged for bacterial distribution by fluorescent microscopy, fixed, and stained in Oil Red O $(0.5 \% \mathrm{w} / \mathrm{v}$ in propylene glycol). Oil Red O density was calculated by using the "measure" function in Image J and subtracting the mean brightness of a representative region within each granuloma from the mean brightness of a representative adjacent "background" region.

\section{Statistics}

All $t$ tests were unpaired $t$ tests with Welch's correction. All analysis of variance (ANOVA) were ordinary one-way ANOVA, comparing the mean of each group with the mean of every other group, using Turkey's multiple comparisons test with a single pooled variance. In cases in which data were pooled from multiple experiments, data from each were normalized to its own within-experiment control (usually "DMSO") before pooling. Outliers were removed using ROUT, with $\mathrm{Q}=1 \%$.

\section{RESULTS}

Mycobacterium marinum Infection Induces Thrombocytosis in Zebrafish To determine whether $M$ marinum induces thrombocytosis in zebrafish, we infected $\operatorname{Tg}(c d 41: G F P)$ embryos, where thrombocytes are marked by GFP expression, with red fluorescent $M$ marinum. Infected embryos had significantly increased density of thrombocytes around the tail venous plexus where granulomas preferentially form (Figure $1 \mathrm{~A}$ and B). Among infected embryos, there was a strong positive correlation between thrombocyte density and mycobacterial burden (Figure 1C). 
A

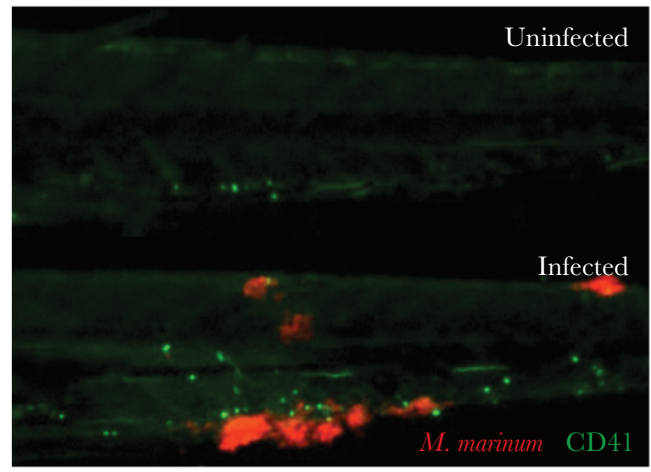

G

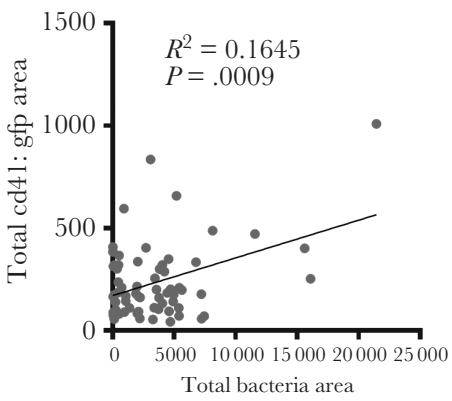

$\mathrm{D}$
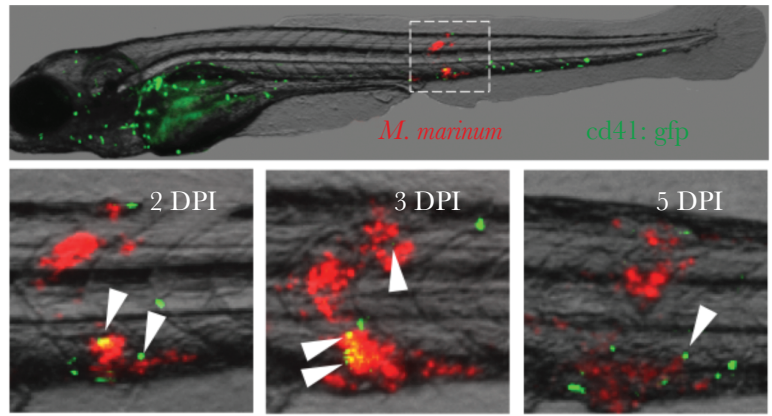

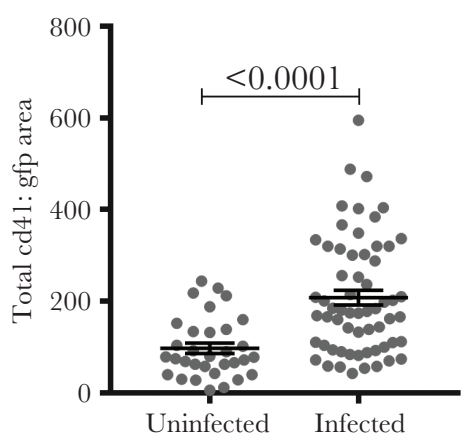

Figure 1. Mycobacterium marinum induces coagulation and thrombosis around sites of infection in zebrafish. (A) Representative images of 5 days postinfection (DPI) Tg(cd41:GFP) thrombocyte lineage reporter embryos infected with M marinum-tdTomato, showing thrombocyte accumulation at sites of bacterial infection. (B) Quantification of total thrombocyte Tglcd41:GFP) area within the tail of uninfected or wild-type M marinum-tdTomato-infected embryos. Graph shows mean \pm standard error of the mean. Statistical analyses performed by $t$ tests. (C) Correlation between M marinum bacterial burden and total thrombocyte Tg(cd41:GFP) area within the tail of infected embryos. $P$ and $\mathrm{R}^{2}$ calculated using linear regression. (D) Representative $2 \mathrm{DPI}, 3 \mathrm{DPI}$, and $4 \mathrm{DPI}$ images of $\mathrm{Tg}(\mathrm{cd} 4 \mathrm{1}$ :GFP) thrombocyte lineage reporter embryos infected with $M$ marinumtdTomato at 3 DPF. White arrowheads show thrombocyte association with areas of bacterial growth. Thrombocytes not indicated with an arrowhead were circulating and not considered to be associated with bacteria.

Because the $c d 41$ promoter is active in nonmotile thrombocyte precursors within their caudal hematopoietic tissue, we could not conclusively determine whether these thrombocytes had actively migrated to and been retained at the site of infection [11]. To determine whether zebrafish thrombocytes are recruited to sites of mycobacterial infection, we performed trunk injection of $M$ marinum in $\mathrm{Tg}(\mathrm{cd} 41$ :GFP) at 3 days postfertilization (DPF), a time point after which mature thrombocytes are in the circulation. Embryos were then imaged at 2, 3, and 4 DPI. Rather than forming a stable and growing clot over a period of days, thrombocytes appeared to form transient associations with sites of infection, and new thrombocytes seemed to be retained at sites of infection in different locations each day (Figure 1D). Therefore, we recorded 30-minute videos of infected embryos using a long pass GFP filter to capture bacteria and thrombocytes simultaneously. Thrombocytes were most often observed on the edges of granulomas consistent with the location of granuloma-defining macrophages [19]. They also formed short associations with sites of infection, sometimes lasting only 5-10 minutes (Supplementary Video 1). Therefore, thrombocyte-granuloma interactions appear to be a conserved feature of mycobacterial infection across species.

\section{Antiplatelet Drugs Reduce Mycobacterial Burden in Zebrafish}

It has previously been reported that aspirin has a host-protective effect during TB infection [20-23]. Most of these studies have focused on the fact that aspirin is a broadly acting nonsteroidal anti-inflammatory drug (NSAID) that is known to modulate infection-relevant prostaglandin metabolism [24]. However, aspirin is also a widely used platelet inhibitor, and we theorized that this capacity may also play a role in the drug's effectiveness against TB.

To test this hypothesis, we first confirmed that aspirin's protective effect was seen across species, by treating $M$ marinuminfected embryos with aspirin by immersion. Mycobacterial burden was reduced by approximately $50 \%$ in aspirin-treated embryos (Figure 2A).

To determine whether the antiplatelet effects of aspirin treatment contribute to the reduced mycobacterial burden, we treated $M$ marinum-infected embryos with the platelet-specific, small molecule glycoprotein IIb/IIIa inhibitors, tirofiban or eptifibatide. These drugs do not inhibit platelet activation and degranulation but rather inhibit activated platelets from binding to one another, and to monocytes, via fibrinogen. Treatment with either glycoprotein IIb/IIIa inhibitor phenocopied aspirin 


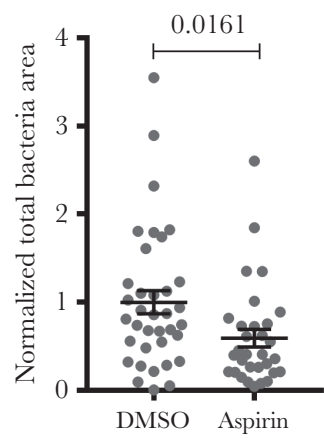

$\mathrm{B}$

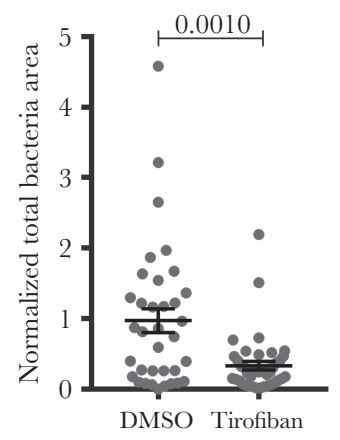

C

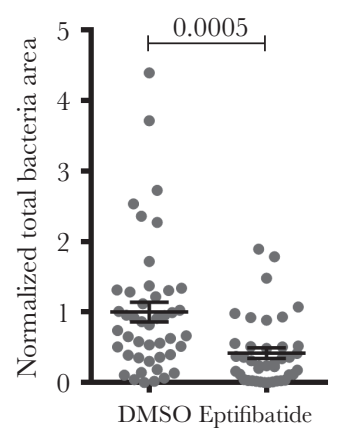

$\mathrm{D}$

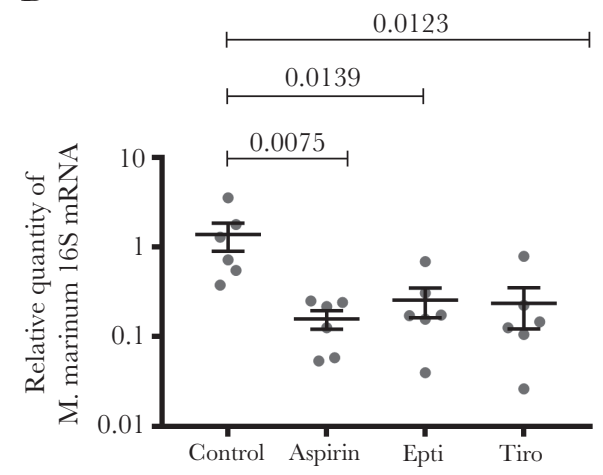

Figure 2. Antiplatelet drugs reduce bacterial burden in Mycobacterium marinum infection. (A) Quantification of bacterial burden in embryos treated with aspirin normalized to dimethyl sulfoxide (DMSO) control. Data are combined results of 2 independent experiments. (B) Quantification of bacterial burden in embryos treated with tirofiban normalized to DMSO control. Data are combined results of 2 independent experiments. (C) Quantification of bacterial burden in embryos treated with eptifibatide or DMSO control. (D) Quantification of bacterial burden in adults treated with aspirin, eptifibatide, or tirofiban, and DMSO control. All graphs show mean \pm standard error of the mean Statistical analyses performed by $t$ tests.

by reducing bacterial burden (Figure $2 \mathrm{~B}$ and $\mathrm{C}$ ). Zebrafish embryos do not have an adaptive immune system; therefore, to determine whether thrombocytes have a similar role in the adaptive response to $M$ marinum, we repeated these drug treatments in adult zebrafish. Aspirin, eptifibatide, and tirofiban reduced bacterial burden in this model (Figure 2D). Taken together, these results provide direct evidence of a pathological role for thrombocyte activation in the immune response to mycobacterial infection.

We next examined the cellular target of antiplatelet drugs in our infection system. We performed antibacterial testing of the antiplatelet drugs in axenic cultures of $M$ marinum and did not observe any effect on bacterial growth in vitro demonstrating host-dependent activity (Supplementary Figure S1A). Treatment of Pseudomonas aeruginosa-infected embryos with either class of antiplatelet drug did not affect survival, demonstrating specificity against mycobacterial infection (Supplementary Figure S1B).

\section{The Antibacterial Effect of Antiplatelet Drugs Is Thrombocyte Dependent} We next confirmed that antiplatelet drugs inhibit thrombocytes in zebrafish, using a tail wound thrombosis assay (Supplementary Figure S2A). Antiplatelet drugs reduced the number of thrombocytes recruited to tail wound clots, demonstrating conservation of their cellular target in zebrafish embryos (Supplementary Figure S2B and C).

To determine whether zebrafish thrombocytes are the conserved target for antiplatelet drugs in the zebrafish- $M$ marinum infection model, we inhibited thrombopoiesis by injection with a morpholino against the thrombopoietin receptor $c m p l$ [11]. Inhibition of thrombopoiesis did not affect the outcome of infection. However, both aspirin and tirofiban treatment failed to reduce bacterial burden in thrombocytedepleted embryos, demonstrating that thrombocytes are the cellular target of this drug in the zebrafish infection model (Figure $3 \mathrm{~A}$ and $\mathrm{B}$ ).

Eptifibatide and tirofiban were designed as specific inhibitors to prevent binding between glycoprotein IIb/IIIa and fibrinogen in mammals [25], but nothing is known about their potential off-target effects in the zebrafish. Therefore, to confirm that disruption of glycoprotein IIb/IIIa binding alone can reduce bacterial burden, we performed infection experiments in glycoprotein IIb/IIIa knockout (KO) transgenic embryos (itga $2 b$ mutants). The itga $2 b^{\text {sa10134 }}$ allele caused a dose-dependent reduction in thrombocytes recruited to tail wound clots (Supplementary Figure S2D), and KO of itga $2 b$ significantly reduced bacterial burden (Figure 3C).

Likewise, when we addressed the same question using a $f$ brinogen alpha chain (fga) mutant zebrafish line that does not produce mature fibrinogen, we saw that KO fish had significantly reduced bacterial burden (Figure 3D). Furthermore, although tirofiban was able to reduce bacterial burden in fgasufficient mutants, it did not reduce bacterial burden in fga KO. Taken together, these data suggest that tirofiban is reducing bacterial burden by inhibiting binding between glycoprotein IIb/IIIa and fibrinogen.

\section{Mycobacterium marinum Induces Coagulation in Zebrafish but Inhibition of Coagulation Does Not Affect the Outcome of Infection}

Seeing that there was a marginal decrease in bacterial burden in $\mathrm{fga}^{-/-}$embryos compared with WT and heterozygous clutchmates, we next sought to determine whether $M$ marinum induces coagulation in zebrafish and the role of coagulation in $M$ marinum infection. We infected $T g(f a b p 10 a: f g b-E G F P)$ transgenic embryos expressing enhanced green fluorescent protein (EGFP)-tagged fibrinogen beta (FGB) with $M$ marinum-tdtomato and imaged the developing infection every 15 minutes from 3 DPI until 6 DPI (Supplementary Video 2). We observed that clots formed only at areas of bacterial growth and that the size of the clots increased 
A

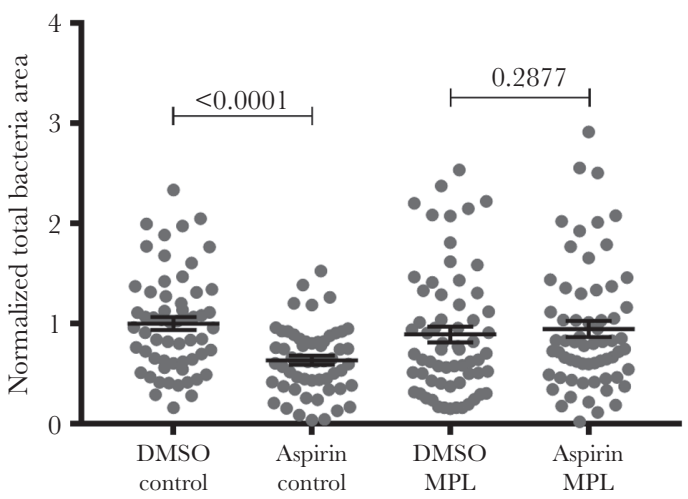

G

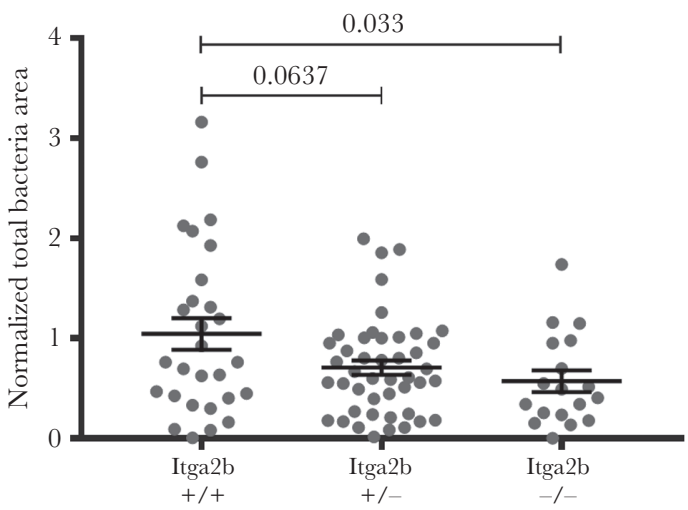

B

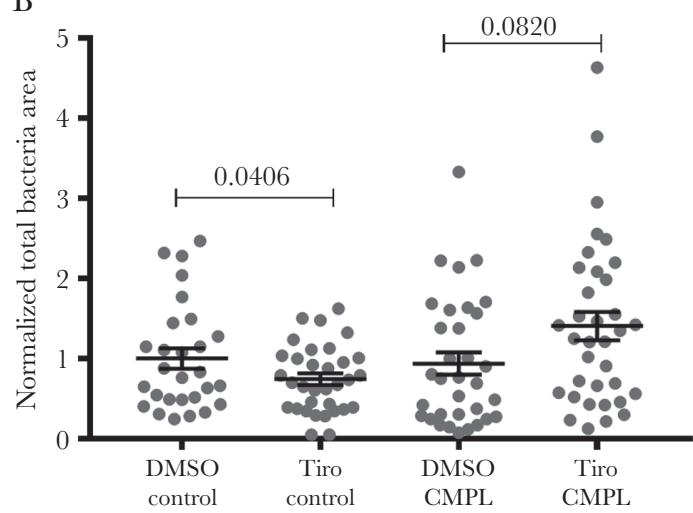

$\mathrm{D}$

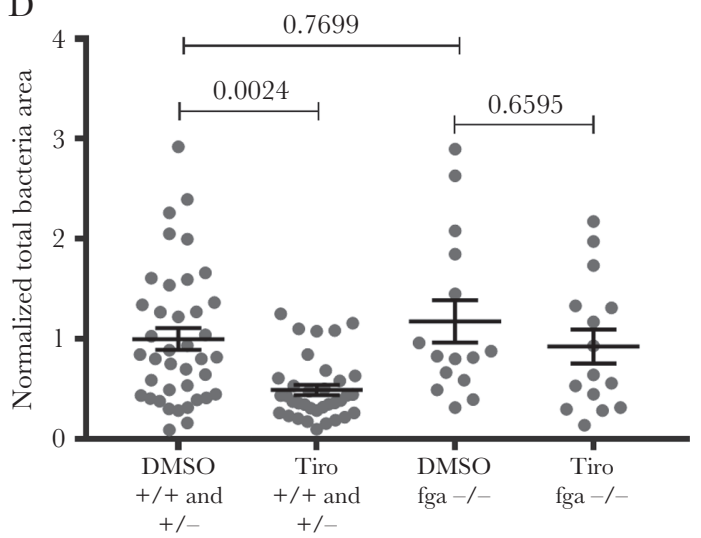

Figure 3. The antibacterial effect of antiplatelet drugs is thrombocyte dependent. (A) Total fluorescence area of Mycobacterium marinum bacteria in larvae injected with either control or CMPL morpholino (MO) to deplete thrombocytes and then treated with aspirin. Values are normalized to dimethyl sulfoxide (DMSO)-treated control M0 larvae. Graphs show the combined results of 2 independent experiments. (B) Total fluorescence area of $M$ marinum bacteria in larvae injected with either control or CMPL MO to deplete thrombocytes and then treated with tirofiban (Tiro). Values are normalized to DMSO-treated control MO larvae. Graphs show the combined results of 2 independent experiments. (C) Quantification of bacterial burden in in itga2b mutant embryos normalized to wild-type control. Data are combined results of 3 independent experiments. (D) Quantification of bacterial burden in $f g a-{ }_{-}^{-}$embryos treated with tirofiban, normalized to $\mathrm{fga}^{+/+}$and $\mathrm{fga}^{+/}$controls. Data are combined results of 2 independent experiments. All graphs show mean \pm standard error of the mean. Statistical analyses performed by $t$ tests or analysis of variance where appropriate.

as the number of bacteria increased over the course of the infection (Figure 4A). When we infected fish with $\triangle \mathrm{ESX} 1$ mutant $M$ marinum, which lacks the ability to export key virulence proteins and does not form granulomas, we observed significantly reduced clot formation (Figures 4B). However, treatment with the anticoagulant warfarin to prevent clot formation during infection with WT $M$ marinum did not affect bacterial burden, suggesting that coagulation itself does not affect bacterial growth within the host (Figure 4C and D). Taken together, these data demonstrate that although coagulation is a consequence of infection driven by a conserved mycobacterial pathogenicity program across host species, the effects of coagulation on mycobacterial pathogenesis may vary between host species [6].

\section{Thrombocytes Increase Mycobacterial Burden Independently of Coagulation}

To assess the contribution of thrombocytes to infection-induced coagulation, we analyzed the formation of FGB-GFP clots in tirofiban-treated embryos. Tirofiban reduced total clot formation
(Figure 5A). However, correction for relative bacterial burden suggested that the reduced clot formation was burden-dependent and thrombus formation was not additionally impacted by tirofiban treatment (Figure 5B). Therefore, we hypothesized that tirofiban was reducing bacterial burden independently of infection-induced coagulation. To investigate this hypothesis, we again used warfarin, which prevented clot formation during infection and did not affect bacterial burden (Figures $4 \mathrm{C}$ and D and $5 \mathrm{C}$ ). As expected, the addition of warfarin to our tirofiban treatment model had no effect on the ability of tirofiban to reduce bacterial burden (Figure 5D), indicating that tirofiban acts through an independent process. This suggests that the protective effect of tirofiban occurs independently of fibrin clot formation, but it requires the presence of soluble fibrinogen.

\section{Thrombocytes Compromise Immunity Through Physical Interactions With Granuloma Macrophages}

To investigate the effect of glycoprotein IIb/IIIa inhibitors on thrombocyte-granuloma interactions, we first measured the 

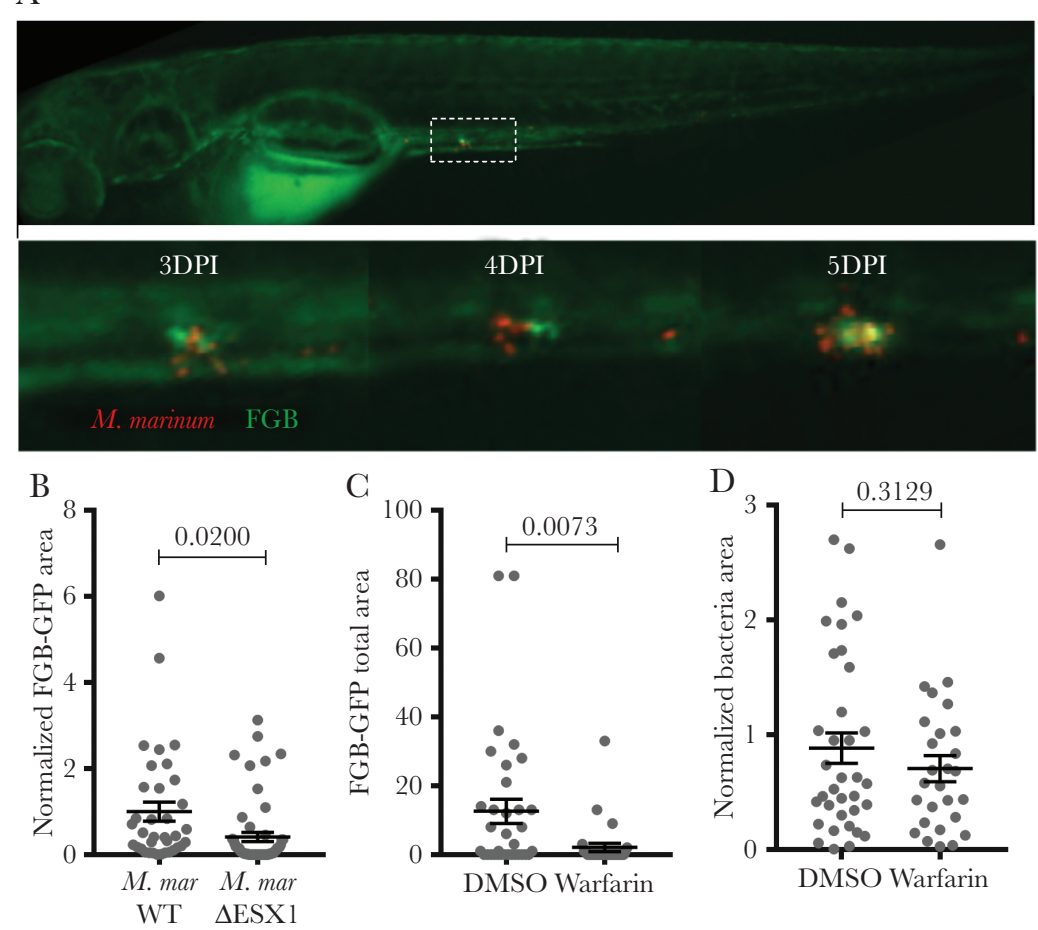

Figure 4. Mycobacterium marinum induces coagulation in zebrafish, but inhibition of coagulation does not affect the outcome of infection. (A) Representative images of a Tg(fabp10a:fgb-EGFP) embryo infected with M marinum-tdTomato by caudal vein injection, showing clot formation at sites of infection at 3, 4, and 5 days postinfection (DPI). (B) Quantification of clot formation in burden-matched $\triangle \mathrm{ESX1}$ mutant-infected Tg(fabp10a:fgb-EGFP) embryos normalized to wild-type (WT) M marinum control. Data show combined results from 2 independent experiments. (C) Quantification of clotting in warfarin-treated Tg(fabp10a:fgb-EGFP) embryos. (D) Quantification of bacterial burden in warfarin-treated embryos, normalized to dimethyl sulfoxide (DMSO) control. Data show combined results of 2 independent experiments. All graphs show mean \pm standard error of the mean. Statistical analysis performed by $t$ test.

effect of tirofiban on the infection-induced thrombocytosis phenotype. We were surprised to find that tirofiban treatment increased thrombocytosis compared with the untreated infected group (Figure 6A). Increased thrombocyte density was also observed in infections with $\triangle \mathrm{ESX} 1$ mutant $M$ marinum, which are unable to drive granuloma maturation or necrosis (Supplementary Figure S2A), yet tirofiban treatment did not reduce $\triangle$ ESX1 M marinum burden (Supplementary Figure S2B), further suggesting that the infection-induced thrombocytosis phenotype is independent of granuloma immunity.

Given that glycoprotein IIb/IIIa-mediated platelet-monocyte binding can occur via fibrinogen $[26,27]$ and patients with pulmonary ТB have been shown to have significantly increased platelet-monocyte aggregation [28], we next aimed to determine whether antiplatelet drugs disrupted the interaction of thrombocytes and macrophages in our infection model. We infected double transgenic $\mathrm{Tg}(\mathrm{cd} 41: G F P$; mfap4:tdtomato) with $M$ marinum-cerulean and performed time-lapse imaging of 5 DPI embryos. Thrombocytes formed transient associations with M marinum-infected macrophages (Supplementary Video 3 ), the duration of which were almost doubled upon glycoprotein IIb/IIIa inhibitor treatment (Supplementary Video 4), with an average dwell time of 20 minutes in eptifibatide-treated embryos and 13 minutes in tirofiban-treated embryos, compared with 11.5 and 7.5 minutes in their respective controls (Figure 6B and C). This suggests that blocking glycoprotein IIb/ IIIa significantly alters the ability of thrombocytes to bind granuloma macrophages.

To demonstrate that thrombocyte inhibition exerts a protective effect through boosting macrophage-dependent immunity, we depleted macrophages by injecting clodronate liposomes to deplete macrophages early during granuloma formation at 3 DPI (Supplementary Figure S3C). Macrophage-depleted fish were unresponsive to tirofiban treatment, demonstrating that pathological thrombocyte activation promotes bacterial growth via interactions with macrophages (Figure 6D). Taken together, these results demonstrate that glycoprotein IIb/IIIa inhibitor treatment disrupts pathological thrombocyte-macrophage attachments in mycobacterial granulomas.

Granuloma Maturation and Pathological Thrombocyte Activation Have a Bidirectional Relationship

Our observation that infection with $\triangle \mathrm{ESX} 1$ mutant $M$ marinum did not result in pathological thrombocyte activation (Supplementary Figure S3A and B) suggested the existence of a bidirectional relationship between granuloma maturation, 


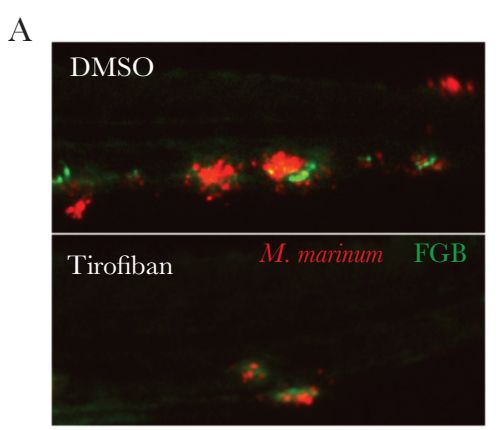

B
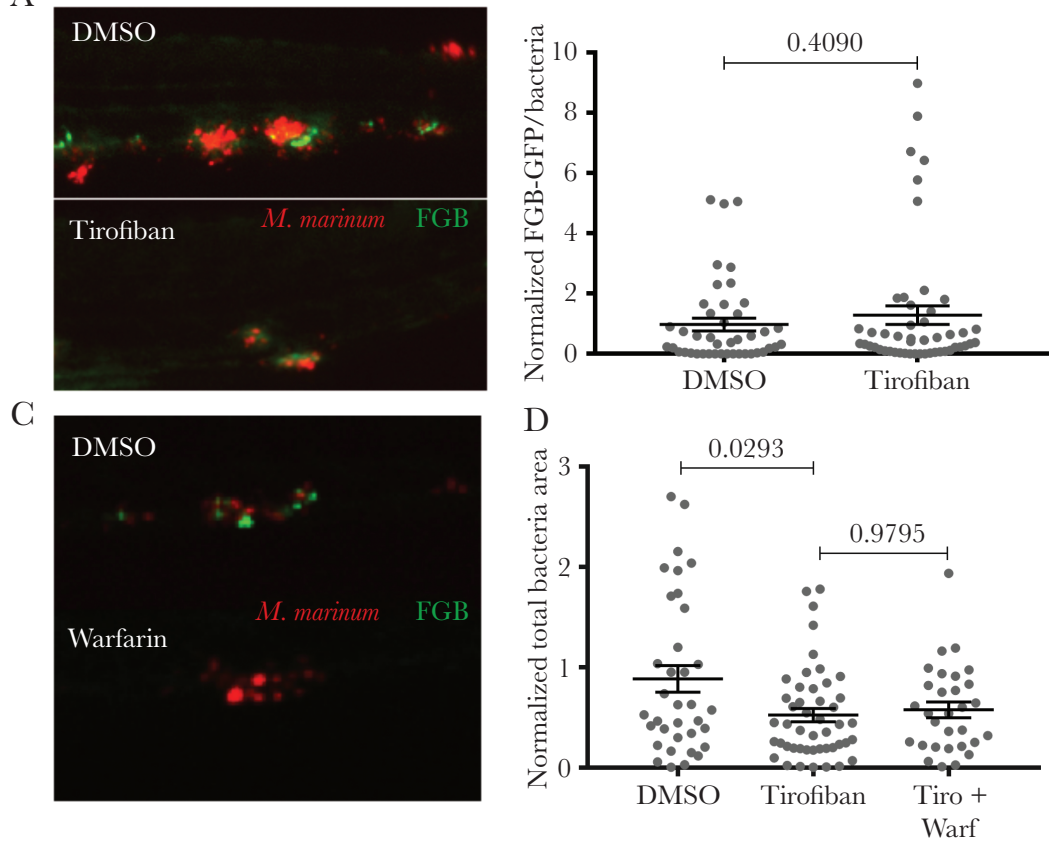

Figure 5. Thrombocytes increase mycobacterial burden independently of coagulation. (A) Representative images of 5 days postinfection (DPI) Tg(fabp10a:fgb-EGFP) embryos infected with Mycobacterium marinum-tdTomato and treated with either dimethyl sulfoxide (DMSO) or tirofiban. (B) Quantification of clotting relative to bacterial burden in embryos treated with tirofiban normalized to DMSO control. Data are combined results of 2 independent experiments. (C) Representative images of Tg/fabp10a:fgbEGFP) embryos, where clot formation can be visualized by green fluorescent protein (GFP), infected with M marinum-tdTomato, and treated with either DMSO or warfarin. (D) Quantification of bacterial burden in embryos treated with tirofiban, warfarin, or tirofiban and warfarin, normalized to DMSO control. Data are combined results of 2 independent experiments. All graphs show mean \pm standard error of the mean. Statistical analysis performed by $t$ test or analysis of variance where appropriate.

which is deficient in $\triangle \mathrm{ESX} 1$ mutant infections, and pathological thrombocyte activation. To further delineate this relationship, we next took advantage of the stereotypical progression of innate immune granulomas in zebrafish embryos and used the burden-reducing effect of tirofiban as a surrogate marker for thrombocyte activation. We found that at 3 DPI, a time point with nascent granuloma formation but before significant granuloma organization and necrosis, tirofiban had no effect on bacterial burden (Supplementary Figure S4A). In contrast, treatment of established infections from 4 to $5 \mathrm{DPI}$, a time point when granulomas become organized and necrotic, tirofiban significantly reduced bacterial burden within 24 hours (Supplementary Figure S4B). Taken together, these data demonstrate the existence of a switch point in granuloma maturity when thrombocytes are either activated or the activation of thrombocytes becomes pathological.

Coincident with the appearance of granuloma necrosis at 4 to $5 \mathrm{DPI}$, we have demonstrated the appearance of foam cells in zebrafish embryo granulomas at this stage of infection [18]. It has previously been shown that platelets accelerate the conversion of macrophages to foam cells in the presence of mycobacteria in vitro [9]. Foam cells are permissive for mycobacterial growth, suggesting a mechanism for infection-induced thrombocyte activation to compromise innate immunity. Therefore, we hypothesized that thrombocyte inhibition would reduce the conversion of macrophages into foam cells. We investigated this by performing Oil red $\mathrm{O}$ staining to measure lipid accumulation within size-matched granulomas (Figure 7A). Tirofiban-treated embryos had significantly less Oil red $\mathrm{O}$ accumulation in their granulomas compared with DMSO control, even after correction for reduced bacterial burden (Figure 7B). Taken together, these data demonstrate an in vivo effect of thrombocyte activation inhibiting an effective immune response by converting macrophages into foam cells in the maturing mycobacterial granuloma.

Given that foam cell formation is closely associated with necrosis in TB [29], we hypothesized that tirofiban treatment would reduce cell death within the granuloma. Therefore, we used TUNEL staining to detect the fragmented deoxyribonucleic acid of dying cells in $M$ marinum-infected embryos. At 5 DPI, tirofiban-treated embryos showed significantly less TUNEL staining, indicating significantly reduced cell death within the granuloma (Figure 7C and D). Taken together, these results indicate that infection-induced thrombocyte activation aggravates pathological markers of granuloma maturation and compromises immune control of mycobacterial infection.

\section{DISCUSSION}

In this study, we used the zebrafish- $M$ marinum model to identify thrombocyte activation as a detrimental host response that 

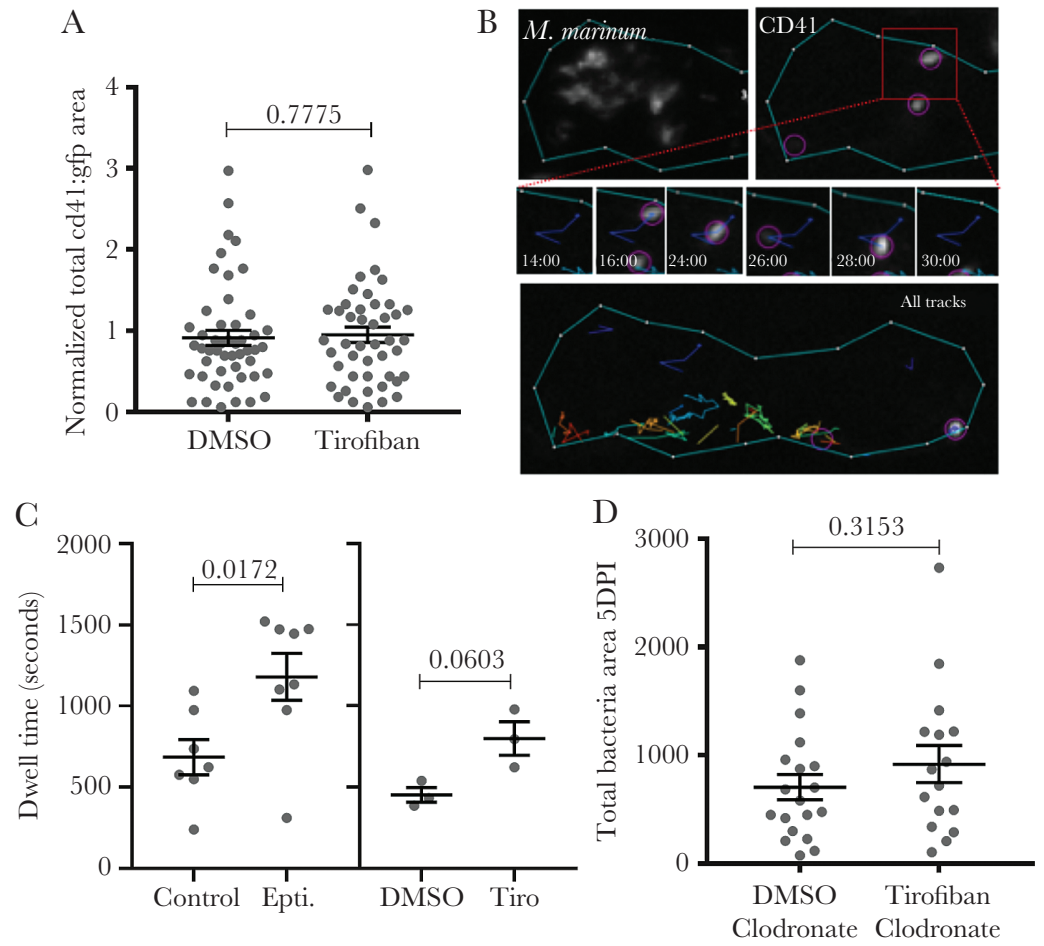

Figure 6. Thrombocyte activation compromises immunity through granuloma-associated macrophages. (A) Quantification of total thrombocyte Tglcd41:GFP) area within the tail wild-type Mycobacterium marinum-tdTomato-infected embryos treated with either dimethyl sulfoxide (DMSO) or tirofiban. (B) Representative image of thrombocyte tracking analysis. A region of interest (ROI) was drawn around areas of $M$ marinum growth and overlaid on CD41 channel. All CD41-positive cells that stayed within the $\mathrm{ROI}$ for a minimum of 2 frames (4 minutes) were tracked, and the time they remained within the ROI was calculated. (C) Quantification of (B), showing average time that thrombocytes remained associated with a site of bacterial accumulation at 5 days postinfection (DPI). Drug treatment was started at 4 DPI and was continued during time lapse. Data points represent average thrombocyte dwell time from each infected fish. Individual fish had from 10 to 50 events recorded. (D) Quantification of bacterial burden in embryos infected with M marinum-tdTomato, injected with clodronate liposomes, and treated with tirofiban at $3 \mathrm{DPI}$. All graphs show mean \pm standard error of the mean. Statistical tests performed by $t$ tests.

is co-opted by pathogenic mycobacteria. Our data builds on previous studies that have shown that coagulation, thrombocytosis, and thrombocyte activation are associated with mycobacterial infection and provide in vivo evidence of a direct role for thrombocyte activation in promoting mycobacterial growth. We showed that infection-induced hemostasis is conserved in the zebrafish-M marinum infection model and that the plateletinhibiting drugs-aspirin, tirofiban, and eptifibatide-are able to reduce bacterial burden through host-mediated effects, independently of effects on coagulation.

Several studies have investigated aspirin as a possible adjunctive treatment for TB in a range of animal models and human trials $[20-24,30,31]$. The results of these studies have been far from conclusive, although most found beneficial effects [20-22, $24,30]$, one human trial observed no effect [23], and a mouse study identified an antagonistic relationship between aspirin and the frontline antitubercular drug isoniazid [31]. This lack of consensus may be because the NSAID effect of aspirin will affect many cell types and processes important in the heterogeneous host response to mycobacterial infection. Our study expands this literature by delineating a role for glycoprotein IIb/ IIIa in compromising the host response to infection.
Our study found that coagulation, thrombocytosis, and thrombocyte activation have distinct roles during the pathogenesis of mycobacterial infection of zebrafish. Inhibiting coagulation alone did not significantly reduce bacterial burden, and therefore we considered antiplatelet treatment as a more attractive HDT. It must be noted that we only measure a simple, single end point in our zebrafish embryo experiments (bacterial load) at a relatively early time point for a chronic infection. In more complex animals, where stroke and DVT are important secondary complications of mycobacterial infection, reducing coagulation may yet prove to be efficacious as an HDT during TB therapy to reduce morbidity. In contrast, data from the mouse model of TB suggests that tissue factor-induced fibrin is necessary to contain mycobacteria within granulomas [32].

Our study provides evidence that although infectioninduced thrombopoiesis is a conserved function of infection, pathological thrombocyte activation is only driven by pathogenic mycobacteria during macrophage-dependent granuloma formation. Our experiments with $\triangle \mathrm{ESX} 1$ mutant $M$ marinum (which cannot secrete key virulence proteins that drive granuloma formation) and clodronate macrophage depletion demonstrated growth restriction by platelet-inhibiting drugs 
A

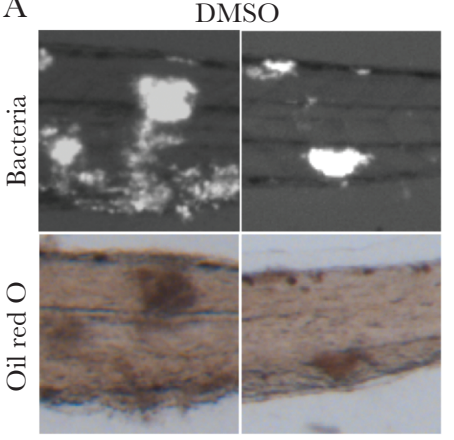

C

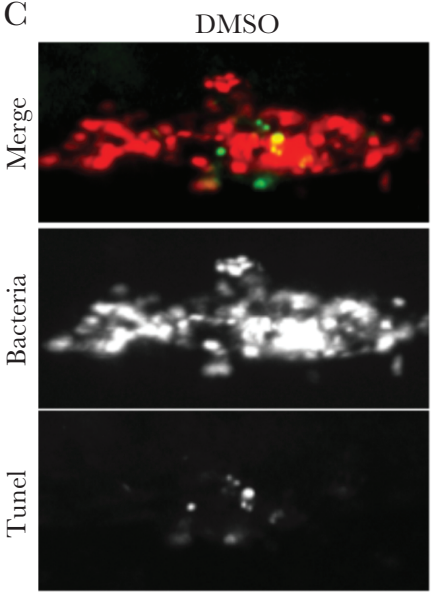

Tirofiban

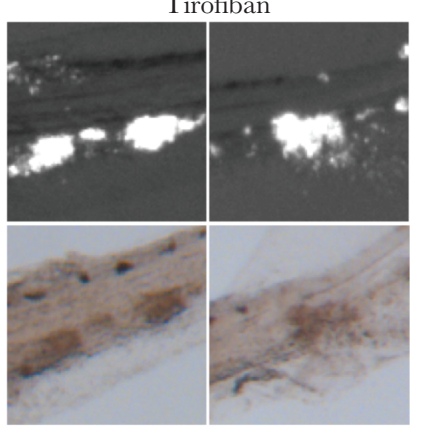

Tirofiban
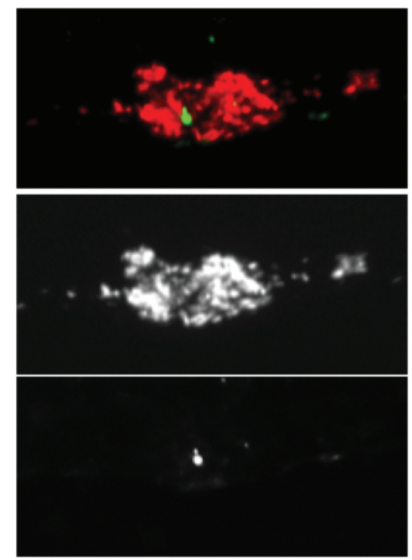

B

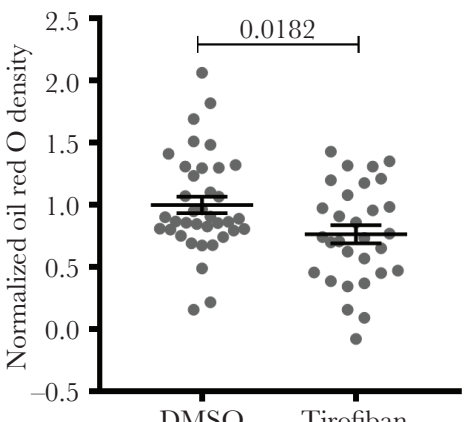

DMSO Tirofiban

D

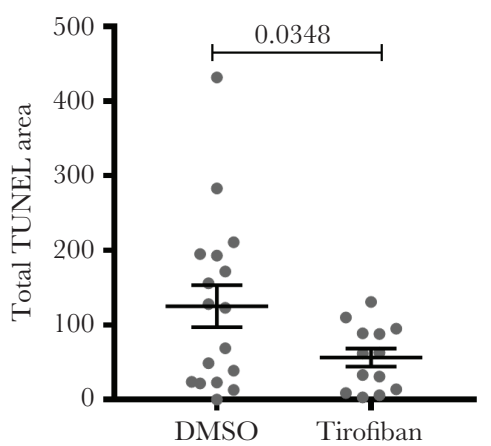

Figure 7. Granuloma maturation and pathological thrombocyte activation have a bidirectional relationship. (A) Representative images of bacterial granulomas chosen for analysis (bacteria are white in grayscale images) and corresponding Oil Red O (ORO) staining (red-brown in online color images). (B) Quantification of ORO pixel density relative to granuloma bacterial area, in embryos treated with tirofiban, normalized to dimethyl sulfoxide (DMSO) control. (C) Representative images of $M$ marinum-tdTomato granulomas and TUNEL Alexafluor-488 staining. (D) Quantification of TUNEL-positive area within the largest granuloma of individual embryos. All graphs show mean \pm standard error of the mean. Statistical tests performed by $t$ tests. Data are combined results of 2 independent experiments, except (D), which represents a single experiment.

is both ESX1 and macrophage dependent. These data fit well with our observations that stationary thrombocytes were only observed around well developed mycobacterial granulomas, and platelet inhibition was only effective at reducing bacterial burden after the development of significant granuloma pathology.

Our imaging and experimental data add evidence that for mammalian platelets, activated thrombocytes can form complexes with leukocytes through fibrinogen binding to glycoprotein IIb/IIIa, and that this alters immune cell function [28, 33-35]. Recent research has highlighted the important role of platelets as innate immune cells; they are able to release antimicrobial peptides, pick up and "bundle" bacteria, and initiate the recruitment of other innate immune cells to sites of infection [36-38]. Our experimental results add to the growing body of literature demonstrating a detrimental effect for platelets in the context of mycobacterial infection.

Platelets can induce macrophages to produce less proinflammatory tumor necrosis factor and interleukin (IL)-1 $\beta$ and more anti-inflammatory IL-10 in response to both Bacillus Calmette-Guérin and $M$ tuberculosis [7, 9, 28]. Crucially, it has been shown that platelets accelerate the formation of foam cells in the context of mycobacterial infection and atheroma [9]. Our experiments demonstrating antiplatelet drug-mediated control of infection and reduced lipid accumulation and cell death in the granuloma suggest that infection-induced thrombocyte activation can be therapeutically modulated in mycobacterial infection.

\section{CONCLUSIONS}

Our findings that inhibition of thrombocyte activation leads to reduced bacterial burden provide important in vivo experimental evidence that infection-induced thrombocyte activation is a potential target for TB HDT. Our experimental demonstration of a thrombocyte-mediated effect of aspirin identifies a novel mechanism by which this well tolerated and low-cost drug may benefit TB patients.

\section{Supplementary Data}

Supplementary materials are available at The Journal of Infectious Diseases online. Consisting of data provided by the authors to benefit the reader, the posted materials are not copyedited 
and are the sole responsibility of the authors, so questions or comments should be addressed to the corresponding author.

\section{Notes}

Author contributions. $\quad$ E. H., D. M. T., and S. H. O. designed the experiments. E. H., K. E. J., M. D. J., T. N., and S. H. O. performed the experiments. J. A. S. generated transgenic and mutant zebrafish lines. E. H. and S. H. O. wrote the paper. W. J. B., D. M. T., and S. H. O. supervised the project.

Acknowledgments. We thank the following: Dr. Kristina Jahn and Sydney Cytometry for assistance with imaging equipment; Garvan Biological Testing Facility staff Jennifer Brand, Michael Pickering, Rola Bazzi, Dr. Lucie Nedved, and Dr. Stephanie Allison at the Garvan Institute of Medical Research for maintenance of zebrafish breeding stock; and Lalita Ramakrishnan, Shaun Jackson, Georges Grau, Carl Feng, and Drs. Jorn Coers and Imala Alwis for helpful discussion of results.

Financial support. This work was funded by the Australian National Health and Medical Research Council (Grant Numbers APP1099912 and APP1053407), University of Sydney Fellowship (Grant Number G197581), NSW Ministry of Health under the NSW Health Early-Mid Career Fellowships Scheme (Grant Number H18/31086), Kenyon Family Inflammation Award 2017 (to S.H.O.), Duke Summer Research Opportunities Program 2015 (to K. E. J.), National Institutes of Health Director's New Innovator Award (Grant Number 1DP2-OD008614; to D. M. T.), and National Institutes of Health (Grant Numbers R01-HL124232 and R01-HL125774; to J. A. S.).

Potential conflicts of interest. All authors: No reported conflicts of interest. All authors have submitted the ICMJE Form for Disclosure of Potential Conflicts of Interest.

\section{References}

1. World Health Organization. Global Tuberculosis Report 2018. World Health Organization 2018.

2. Oehlers SH, Cronan MR, Scott NR, et al. Interception of host angiogenic signalling limits mycobacterial growth. Nature 2015; 517:612-5.

3. Oehlers SH, Cronan MR, Beerman RW, et al. Infectioninduced vascular permeability aids mycobacterial growth. J Infect Dis 2017; 215:813-7.

4. Robson SC, White NW, Aronson I, Woollgar R, Goodman H, Jacobs P. Acute-phase response and the hypercoagulable state in pulmonary tuberculosis. Br J Haematol 1996; 93:943-9.

5. Kutiyal AS, Gupta N, Garg S, Hira HS. A study of haematological and haemostasis parameters and hypercoagulable state in tuberculosis patients in northern India and the outcome with anti-tubercular therapy. J Clin Diagn Res 2017; 11:OC09-13.
6. Kothari H, Rao LV, Vankayalapati R, Pendurthi UR. Mycobacterium tuberculosis infection and tissue factor expression in macrophages. PLoS One 2012; 7:e45700.

7. Fox KA, Kirwan DE, Whittington AM, et al. Platelets regulate pulmonary inflammation and tissue destruction in tuberculosis. Am J Respir Crit Care Med 2018; 198:245-55.

8. Dong Z, Shi J, Dorhoi A, et al. Hemostasis and lipoprotein indices signify exacerbated lung injury in $\mathrm{TB}$ with diabetes comorbidity. Chest 2018; 153:1187-200.

9. Feng Y, Dorhoi A, Mollenkopf HJ, et al. Platelets direct monocyte differentiation into epithelioid-like multinucleated giant foam cells with suppressive capacity upon mycobacterial stimulation. J Infect Dis 2014; 210:1700-10.

10. Vo AH, Swaroop A, Liu Y, Norris ZG, Shavit JA. Loss of fibrinogen in zebrafish results in symptoms consistent with human hypofibrinogenemia. PLoS One 2013; 8:e74682.

11. Lin HF, Traver D, Zhu H, et al. Analysis of thrombocyte development in CD41-GFP transgenic zebrafish. Blood 2005; 106:3803-10.

12. Walton EM, Cronan MR, Beerman RW, Tobin DM. The macrophage-specific promoter mfap4 allows live, longterm analysis of macrophage behavior during mycobacterial infection in zebrafish. PLoS One 2015; 10:e0138949.

13. Hu Z, Lavik KI, Liu Y, et al. Loss of fibrinogen in zebrafish results in an asymptomatic embryonic hemostatic defect and synthetic lethality with thrombocytopenia. J Thromb Haemost 2019; 17:607-17. doi:0.1111/jth.14391.

14. Walton EM, Cronan MR, Cambier CJ, et al. Cyclopropane modification of Trehalose dimycolate drives Granuloma angiogenesis and mycobacterial growth through VEGF signaling. Cell Host Microbe 2018; 24:514-525.e6.

15. Matty MA, Oehlers SH, Tobin DM. Live imaging of hostpathogen interactions in zebrafish larvae. Methods Mol Biol 2016; 1451:207-23.

16. Tinevez JY, Perry N, Schindelin J, et al. TrackMate: an open and extensible platform for single-particle tracking. Methods 2017; 115:80-90.

17. Passeri MJ, Cinaroglu A, Gao C, Sadler KC. Hepatic steatosis in response to acute alcohol exposure in zebrafish requires sterol regulatory element binding protein activation. Hepatology 2009; 49:443-52.

18. Johansen MD, Kasparian JA, Hortle E, Britton WJ, Purdie AC, Oehlers SH. Mycobacterium marinum infection drives foam cell differentiation in zebrafish infection models. Dev Comp Immunol 2018; 88:169-72.

19. Cronan MR, Beerman RW, Rosenberg AF, et al. Macrophage epithelial reprogramming underlies Mycobacterial granuloma formation and promotes infection. Immunity 2016; 45:861-76.

20. Mai NT, Dobbs N, Phu NH, et al. A randomised double blind placebo controlled phase 2 trial of adjunctive aspirin 
for Tuberculous meningitis in HIV-uninfected adults. Elife 2018; 7:pii: e33478.

21. Kroesen VM, Rodriguez-Martinez P, Garcia E, et al. A beneficial effect of low-dose aspirin in a murine model of active tuberculosis. Front Immunol 2018; 9:798.

22. Misra UK, Kalita J, Nair PP. Role of aspirin in tuberculous meningitis: a randomized open label placebo controlled trial. J Neurol Sci 2010; 293:12-7.

23. Schoeman JF, Janse van Rensburg A, Laubscher JA, Springer P. The role of aspirin in childhood tuberculous meningitis. J Child Neurol 2011; 26:956-62.

24. Tobin DM, Roca FJ, Oh SF, et al. Host genotype-specific therapies can optimize the inflammatory response to mycobacterial infections. Cell 2012; 148:434-46.

25. Peerlinck K, De Lepeleire I, Goldberg M, et al. MK-383 (L-700,462), a selective nonpeptide platelet glycoprotein IIb/IIIa antagonist, is active in man. Circulation 1993; 88:1512-7.

26. Altieri DC, Mannucci PM, Capitanio AM. Binding of fibrinogen to human monocytes. J Clin Invest 1986; 78: 968-76.

27. Armstrong PC, Kirkby NS, Chan MV, et al. Novel whole blood assay for phenotyping platelet reactivity in mice identifies ICAM-1 as a mediator of platelet-monocyte interaction. Blood 2015; 126:e11-8.

28. Kullaya V, van der Ven A, Mpagama S, et al. Plateletmonocyte interaction in Mycobacterium tuberculosis infection. Tuberculosis (Edinb) 2018; 111:86-93.

29. Russell DG, Cardona PJ, Kim MJ, Allain S, Altare F. Foamy macrophages and the progression of the human tuberculosis granuloma. Nat Immunol 2009; 10:943-8.
30. Byrne ST, Denkin SM, Zhang Y. Aspirin and ibuprofen enhance pyrazinamide treatment of murine tuberculosis. J Antimicrob Chemother 2007; 59:313-6.

31. Byrne ST, Denkin SM, Zhang Y. Aspirin antagonism in isoniazid treatment of tuberculosis in mice. Antimicrob Agents Chemother 2007; 51:794-5.

32. Venkatasubramanian S, Tripathi D, Tucker T, et al. Tissue factor expression by myeloid cells contributes to protective immune response against Mycobacterium tuberculosis infection. Eur J Immunol 2016; 46:464-79.

33. Weber C, Springer TA. Neutrophil accumulation on activated, surface-adherent platelets in flow is mediated by interaction of Mac-1 with fibrinogen bound to alphaIIbbeta3 and stimulated by platelet-activating factor. J Clin Invest 1997; 100:2085-93.

34. Sanderson HM, Fox SC, Robbins RA, Lösche W, Spangenberg P, Heptinstall S. Role of GPIIb-IIIa in plateletmonocyte and platelet-neutrophil conjugate formation in whole blood. Platelets 1998; 9:245-50.

35. Kral JB, Schrottmaier WC, Salzmann M, Assinger A. Platelet interaction with innate immune cells. Transfus Med Hemother 2016; 43:78-88.

36. Gaertner F, Ahmad Z, Rosenberger G, et al. Migrating platelets are mechano-scavengers that collect and bundle bacteria. Cell 2017; 171:1368-1382.e23.

37. Li JL, Zarbock A, Hidalgo A. Platelets as autonomous drones for hemostatic and immune surveillance. J Exp Med 2017; 214:2193-2204.

38. Morrell CN, Aggrey AA, Chapman LM, Modjeski KL. Emerging roles for platelets as immune and inflammatory cells. Blood 2014; 123:2759-67. 